\title{
SHEAR DEFORMATION ANALYSIS ON LASER-INDUCED REVERSE BULGING AND PLUGGING
}

\author{
Y. C. Zhou, ${ }_{\ddagger}^{\ddagger}$ Z. P. Duan $\dagger$ and Q. B. Yang* \\ * Department of Physics, Xiangtan University, 411105, Xiangtan Hunan, \\ Pcople's Republic of China; \\ † Laboratory for Laser and Dynamic Behaviors of Materials, Institute of Mechanics, CAS, Beijing, \\ 100080, People's Republic of China
}

(Received for publication 9 April 1997)

\begin{abstract}
A new kind of failure mode is observed in circular brass foils in which their peripheries are fixed and their surfaces are subjected to a long pulsed laser over a central region. The failure is classified into three stages; they are referred to as thermal bulging, localized shear deformation and perforation by plugging. A distinct feature of the failure mode is that bulging and plugging occurred in the direction opposite to the incident laser beam. To study the failure mode, we investigate the non-linear response of heated, non-homogeneous circular plates. Based on the large deflection equations of Berger [J. Appl. Mech. 22 (3), 465-472 (1965)], Ohnabe and Mizuguchi [Int. J. Non-Linear Mech. 28 (4), 365-372 (1993)] and the parabolic shear deformation theory of Bhimaraddi and Stevens [J. Appl. Mech. 51 (1), 195-198 (1984)], we have derived new coupled governing equations of shear deformation and deflection. The new equations are solved, for the plate with a clamped edge, by the Galerkin and iterative methods. The numerical results for the shear deformation distribution are in good agreement with the experimental observation. (C) 1997 Elsevier Science Ltd.
\end{abstract}

Keywords: laser beam, reverse bulging and plugging, shear deformation

\section{INTRODUCTION}

Interaction of a high power laser with materials leads to two kinds of coupled damage, mechanical and thermal damage, depending on the laser parameters and target material mechanical properties [1-11]. As far as the laser parameters are concerned, the intensity $I$ and the duration $\tau$ are usually considered as the two main governing factors, of which the different values could produce different types of damage $[3,4]$. Depending on the intensity and concentration of a laser beam over a solid, material damage could occur by spallation, melting and/or vaporization [4]. However, in the previous studies on laser-induced material damage, people ignored more or less the fact that the spatial shapes of the laser beam could also play an important role in controlling the failure mode. Those situations prevail where the size and intensity of the laser beam are such that the spatial structure effect will contribute to the mode of failure such as bulging followed by plugging. Such are the cases considered in this investigation.

A new kind of failure mode is observed in circular brass foils in which their peripheries are fixed and their surfaces are subjected to a long pulsed laser over a central region, as shown in Fig. 1. The $z$ - and $r$-direction are aligned normal and parallel to the specimen. The foil has a thickness of $0.1 \mathrm{~mm}$. The spatial distribution of the laser intensity is non-Gaussian, but roughly uniform within the laser irradiated region and declines very sharply towards the edge where the laser spot terminates. Let $2 a_{0}$ denote the diameter of the laser spot that can vary from 2 to $6 \mathrm{~mm}$. Illustrated schematically in Fig. 2 is the evolution of specimen failure [12-14]. Figure 2(a) shows bulging of the brass foil at the earlier stage of laser irradiation. Note that this occurs towards the side of the incident laser beam where the temperature would be higher. Considerable shear deformation occurs around a rim near the outer edge of the laser beam which is shown in Fig. 2(b). This leads to the softening of the material due to intense heating. 


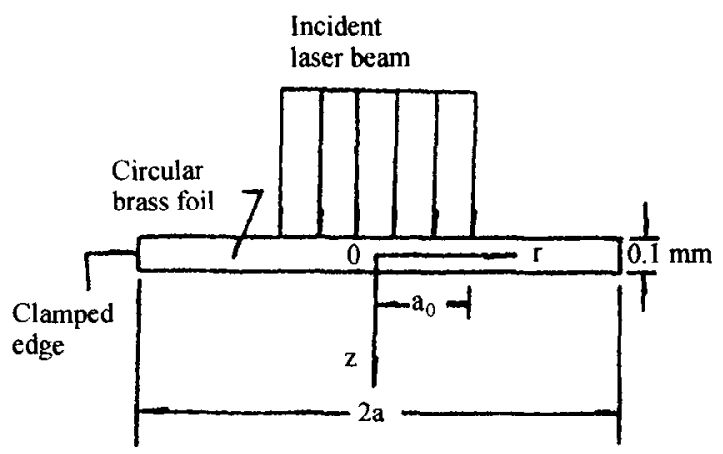

Fig. 1. Schematic of a normal incident laser beam impinging on a circular brass foil specimen.

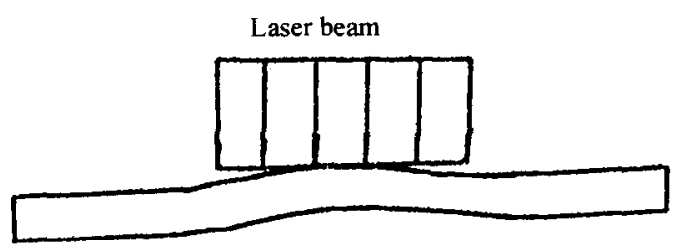

(a) Bulging

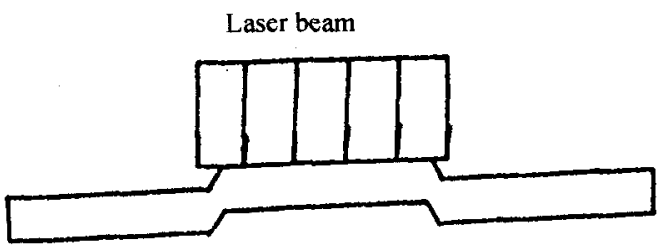

(b) Localized shear deformation

Laser beam

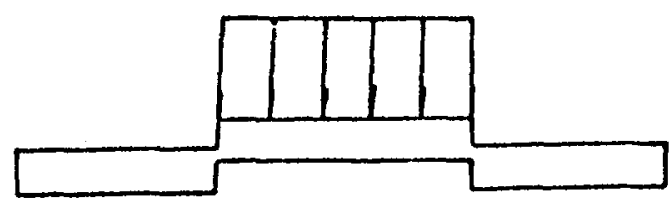

(c) Plugging initiation

Laser beam
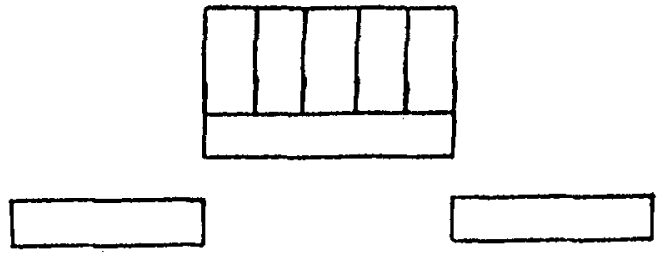

(d) Perforation

Fig. 2. Schematic of damage evolution: (a) bulging; (b) localized shear deformation; (c) plugging initiation and (d) perforation.

Further intensification of the energy around the periphery of the laser beam leads to the initiation of plugging and final perforation which are shown in Fig. 2(c) and (d), respectively. The plugging mode of failure is customarily known to be associated with metal projectiles penetrating through metal targets in plate form $[15,16]$. A plug of the target material is ejected in the direction of the energy source that is the moving projectile. In the case of an incident laser beam, plugging occurred opposite to the incoming direction of the energy 


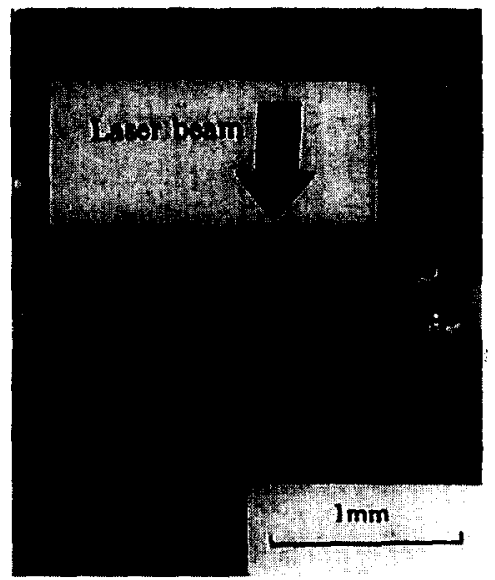

Fig. 3. Photo of sectioned brass foil at the bulge state with a laser energy of $8.2 \mathrm{~J}$ over an area of $2.3 \mathrm{~mm}$ in diameter.

source. The initial bulge occurs on the side with higher temperature that determines the direction of plugging. Figure 3 is the photo of a polished section of brass specimen that shows the target bulging in the direction opposite to the laser beam [13].

To explain the new failure mode, the non-linear response of heated, non-homogeneous circular plates has been considered based on the large deflection equations of Berger [17] and Ohnabe and Mizuguchi [18] and the parabolic shear deformation theory of Bhimaraddi and Chandrashekhara [19] and Bhimaraddi and Stevens [20]. The new nonlinear coupled governing equations are derived for the case of a clamped boundary condition which is restrained from radial movement. The governing equations are solved by using the Galerkin and iterative methods. The numerical results for the shear deformation distribution show that the shear deformation induced by a non-Gaussian type laser beam in the laser spot edge region is much larger than that elsewhere. From the comparison of shear deformation distributions induced by a non-Gaussian and Gaussian type laser beam, one concludes that the distinct feature of the spatial distribution of the laser intensity induces the new kind of failure mode.

\section{NON-LINEAR COUPLED EQUATIONS}

We considered an elastic, heated circular plate of radius $a$ as shown in Fig. 1. Let the non-homogeneity function of the material depend only on the coordinate $r$ and be independent of $\varphi$ and $z$, but strongly vary with temperature. Let the deformation be axisymmetric with respect to the center. Therefore, the terms of the circumferential displacement $v$ and those with respect to $\partial / \partial \varphi$ are eliminated. Following Bhimaraddi and Stevens [20], the displacement components are assumed to be

$$
\bar{u}=u+\xi \phi-z w^{\prime}, \quad \bar{w}=w
$$

where

$$
\xi=z\left(1-\frac{4 z^{2}}{3 h^{2}}\right) \quad \text { and } \quad \xi^{*}=\frac{\mathrm{d} \xi}{\mathrm{d} z}=\left(1-\frac{4 z^{2}}{h^{2}}\right)
$$

In expression (1), $\bar{u}$ and $\bar{w}$ are the displacements of any point $(r, z)$ of the plate of the $r$-and $z$-directions, respectively; $u$ and $w$ are the displacements of any point on the middle surface $(z=0)$ of the plate in the $r$-and $z$-direction, respectively; $\phi$ is the shear rotation of any point on the middle surface of the plate in addition to the flexural rotations $w^{\prime} ; h$ is the total thickness of the plate; ( $)^{\prime}$ indicates differentiation with respect to $r$. It may be seen that $u, w$ and $\phi$ are functions of $r$ only.

It may be noted that the preceding form of expressions for displacement components results in the parabolic variation of transverse shear strains as is evident from equation (5). To limit the complexity of the problem to a reasonable degree, the expression for $w$ is chosen to be a constant and at the same time this allows us to have zero transverse shear strains at 
the top and bottom surfaces of the plate. Using the above displacement forms, the strain-displacement relations relevant to the current study are written as

$$
\begin{aligned}
\varepsilon_{r r} & =u^{\prime}+\xi \phi^{\prime}-z w^{\prime \prime}+\frac{1}{2}\left(w^{\prime}\right)^{2} \\
\varepsilon_{\theta \theta} & =\frac{u}{r}+\xi \frac{\phi}{r}-z \frac{1}{r} w^{\prime} \\
\varepsilon_{r z} & =\frac{1}{2} \xi^{*} \phi
\end{aligned}
$$

The stress and strain relationships are

$$
\begin{aligned}
\sigma_{r r} & =\frac{E}{1-v^{2}}\left[\varepsilon_{r r}+v \varepsilon_{\theta \theta}-(1-v) \alpha \theta\right] \\
\sigma_{\theta \theta} & =\frac{E}{1-v^{2}}\left[\varepsilon_{\theta \theta}+v \varepsilon_{r r}-(1-v) \alpha \theta\right] \\
\tau_{r z} & =\frac{E}{1+v} \varepsilon_{r z}
\end{aligned}
$$

where $E, v, \alpha$ are the Young's modulus, the Poisson ratio and the thermal expansion coefficient which are assumed to be functions of temperature $T$. In the above formulas, $\theta=T-T_{0}$ is the temperature rise, $T$ and $T_{0}$ are the absolute temperature and room temperature, respectively.

The total potential energy $V[21]$ is

$$
\begin{aligned}
V= & 2 \pi \int_{0}^{a} r \mathrm{~d} r \int_{-h / 2}^{h / 2} \mathrm{~d} z\left[\frac{1}{2} \frac{v E}{(1+v)(1-2 v)}\left(\varepsilon_{r r}+\varepsilon_{\theta \theta}+\varepsilon_{z z}\right)^{2}\right. \\
& \left.+\frac{E}{2(1+v)}\left(\varepsilon_{r r}^{2}+\varepsilon_{\theta \theta}^{2}+\varepsilon_{z z}^{2}+2 \varepsilon_{r z}^{2}\right)-\frac{E \alpha \theta}{1-2 v}\left(\varepsilon_{r r}+\varepsilon_{\theta \theta}+\varepsilon_{z z}\right)-\frac{\rho C_{\mathrm{p}}}{2 T_{0}} \theta^{2}\right]
\end{aligned}
$$

where $\rho, C_{\mathbf{p}}$ are the mass density and specific heat capacity of materials, respectively. Due to the thin specimen and the free surface, the stress $\sigma_{z z}$ is assumed to be zero. Therefore, from the following equation

$$
\sigma_{z z}=\frac{E}{1+v} \varepsilon_{z z}+\frac{v E}{(1+v)(1-2 v)}\left(\varepsilon_{r r}+\varepsilon_{\theta \theta}+\varepsilon_{z z}\right)-\frac{E \alpha \theta}{1-2 v}=0
$$

we have

$$
\varepsilon_{z z}=\frac{1+v}{1-v} \alpha \theta-\frac{v}{1-v}\left(\varepsilon_{r r}+\varepsilon_{\theta \theta}\right)
$$

Substituting equations (1)-(8) and (11) in (9), one can obtain the following expression for $V$ :

$$
\begin{aligned}
V= & \pi \int_{-h / 2}^{h / 2} \mathrm{~d} z \int_{0}^{a} r \mathrm{~d} r\left\{\frac { E } { 1 - v ^ { 2 } } \left[\varepsilon_{r r}^{2}+2 v \varepsilon_{r r} \varepsilon_{\theta \theta}+\varepsilon_{\theta \theta}^{2}-2(1+v) \alpha \theta\left(\varepsilon_{r r}+\varepsilon_{\theta \theta}\right)\right.\right. \\
& \left.\left.+2(1-v) \varepsilon_{r z}^{2}\right]-\frac{2(1+v) E}{(1-v)(1-2 v)}(\alpha \theta)^{2}-\frac{\rho C_{\mathrm{p}}}{T_{0}} \theta^{2}\right\} \\
& \pi \int_{0}^{a} r D \mathrm{~d} r\left\{\left(\nabla^{2} w\right)^{2}-\frac{2}{r}(1-v) w^{\prime} w^{\prime \prime}+\frac{12}{h^{2}}\left[\varepsilon_{1}^{2}+2(v-1) \varepsilon_{2}\right]\right. \\
& +\alpha(1+v) \frac{24}{h^{3}}\left[m_{\mathrm{T}} \nabla^{2} w-\varepsilon_{1} p_{\mathrm{T}}-\phi\left(m_{\mathrm{T}}-n_{\mathrm{T}}\right)\right]+\frac{68}{105}\left[\phi^{2}+2 \phi^{\prime} \frac{\phi}{r}(v-1)\right] \\
& -\frac{8}{5}\left[\Phi \nabla^{2} w+(v-1)\left(w^{\prime \prime} \frac{\phi}{r}+\phi^{\prime} \frac{w^{\prime}}{r}\right)\right]+\frac{16}{5} \frac{1}{h^{2}}(1-v) \phi^{2} \\
& \left.-\left[\frac{2(1+v)}{(1-v)(1-2 v)} E \alpha^{2} q_{\mathrm{T}}+\frac{\rho C_{\mathrm{p}}}{T_{0}} q_{\mathrm{T}}\right]\right\}
\end{aligned}
$$


where $D$ is the bending rigidity expressed by

$$
D=\frac{E h^{3}}{12\left(1-v^{2}\right)}
$$

and

$$
\begin{aligned}
m_{\mathrm{T}}=\int_{-h / 2}^{h / 2} \theta z \mathrm{~d} z, & n_{\mathrm{T}}=\frac{4}{3 h^{2}} \int_{-h / 2}^{h / 2} \theta z^{3} \mathrm{~d} z \\
p_{\mathrm{T}}=\int_{-h / 2}^{h / 2} \theta \mathrm{d} z, & q_{\mathrm{T}}=\int_{-h / 2}^{h / 2} \theta^{2} \mathrm{~d} z
\end{aligned}
$$

In expression (13) $\varepsilon_{1}$ and $\varepsilon_{2}$ are the first and second invariant,

$$
\varepsilon_{1}=\bar{\varepsilon}_{r r}+\bar{\varepsilon}_{\theta \theta}, \quad \varepsilon_{2}=\widetilde{\varepsilon}_{r r} \vec{\varepsilon}_{\theta \theta}
$$

where

$$
\overline{\varepsilon_{r r}}=u^{\prime}+\frac{1}{2} w^{\prime 2}, \quad \overline{\varepsilon_{\theta \theta}}=\frac{u}{r}
$$

In equation (13),

$$
\nabla^{2}=\frac{\mathrm{d}^{2}}{\mathrm{~d} r^{2}}+\frac{1}{r} \frac{\mathrm{d}}{\mathrm{d} r}
$$

is the Laplace operator and

$$
\phi=\phi^{\prime}+\frac{\phi}{r}
$$

In expression (13), according to Berger [17] and Ohnabe and Mizuguchi [18] eliminating the second strain invariant $\varepsilon_{2}$ and appling the Euler-Lagrange variational principle for minimum potential energy, the Euler-Lagrange differential equations become the following non-linear coupled governing equations for $u, w$ and $\phi$

$$
\begin{gathered}
\frac{\mathrm{d}}{\mathrm{d} r}\left(D \varepsilon_{1}\right)=\frac{1}{h} \frac{\mathrm{d}}{\mathrm{d} r}\left[D \alpha(1+v) p_{\mathrm{T}}\right] \\
\nabla^{2}\left(D \nabla^{2} w\right)-\frac{12}{h^{2}} D \varepsilon_{1} \nabla^{2} w-\frac{1}{r}\left\{\frac{\mathrm{d}^{2}}{\mathrm{~d} r^{2}}[(1-v) D] w^{\prime}+\frac{\mathrm{d}}{\mathrm{d} r}[(1-v) D] w^{\prime \prime}\right\} \\
+\frac{12}{h^{3}}\left\{D \alpha(1+v)\left(\nabla^{2} m_{\mathrm{T}}+p_{\mathrm{T}} \nabla^{2} w\right)+m_{\mathrm{T}} \nabla^{2}[D \alpha(1+v)]+2 m_{\mathrm{T}}^{\prime} \frac{\mathrm{d}}{\mathrm{d} r}[D \alpha(1+v)]\right\} \\
-\frac{4}{5}\left\{\nabla^{2}(D \Phi)+\phi^{\prime} \frac{\mathrm{d}}{\mathrm{d} r}[D(v-1)]+\phi \frac{\mathrm{d}^{2}}{\mathrm{~d} r^{2}}[D(v-1)]\right\}=0 \\
\frac{\mathrm{d}}{\mathrm{d} r}\left[D \nabla^{2} w+\frac{15}{h^{3}} D \alpha(1+v)\left(m_{\mathrm{T}}-n_{\mathrm{T}}\right)-\frac{17}{21} D \phi\right] \\
-\left(\frac{17}{21} \phi-w^{\prime}\right) \frac{1}{r} \frac{\mathrm{d}}{\mathrm{d} r}[D(v-1)]+\frac{4}{h^{2}}(1-v) D \phi=0
\end{gathered}
$$

The corresponding equations for the Kármán equations can be obtained from the above equations using $\phi=0$ and $D=D_{0}, \alpha=\alpha_{0}, v=v_{0}$, where $D_{0}, \alpha_{0}, v_{0}$ are the corresponding material parameters at room temperature.

\section{THERMAL FIELD AND THE SOLUTION OF GOVERNING EQUATIONS}

\subsection{Thermal field}

Determination of the temperature rise $\theta=T-T_{0}=\theta(r, z, t)$ becomes essential to reveal the mechanism of the new failure mode. Heat from a laser pulse with temporal profile $g(t)$ and radial intensity distribution $f(r)$ is absorbed in the surface plane of the brass foil. 


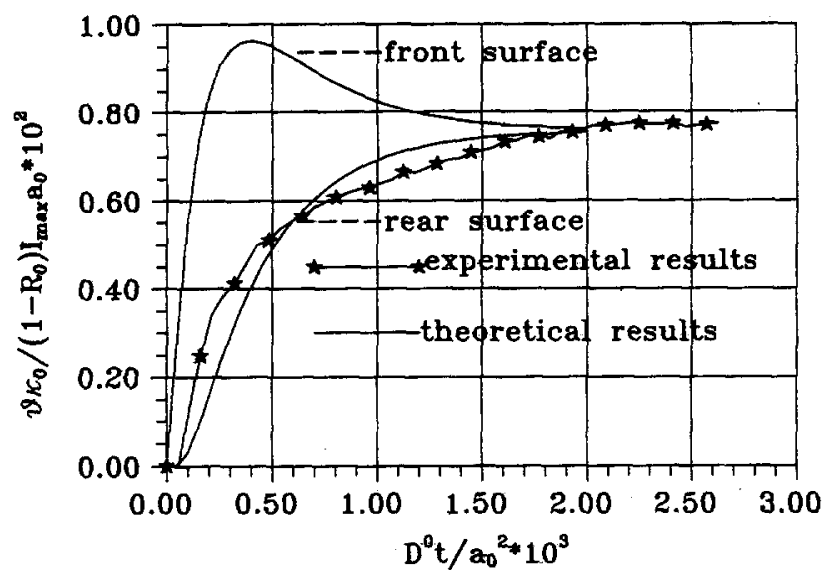

Fig. 4. Histories of the dimensionless temperature rise in the centers of the front and rear surfaces.

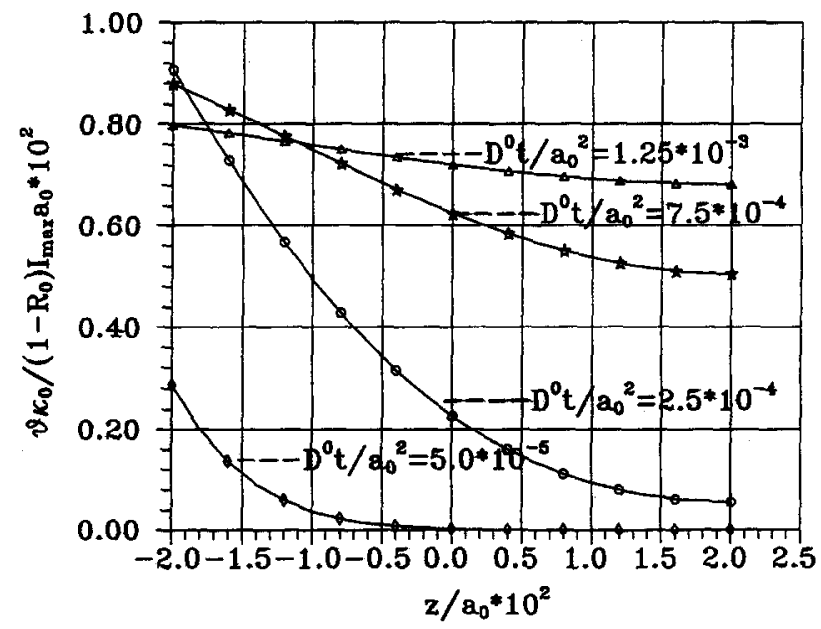

Fig. 5. Variations of dimensionless temperature rise with $z / a_{0}$ in the center of a brass foil.

From the experimental test [12-14], the laser intensity $I$ is approximated by,

$$
I=I_{\max } \mathrm{e}^{-\alpha_{1} t}\left(1-\mathrm{e}^{-\beta_{1} t}\right) f(r)=I_{\max } g(t) f(r)
$$

where $t$ is time, $I_{\max }$ is the maximum value of laser intensity, $\alpha_{1}$ and $\beta_{1}$ are determined experimentally, and equal to $1.5 \times 10^{4}$ and $8.0 \times 10^{4} \mathrm{~s}^{-1}$, respectively. Therefore, laser energy $E_{\mathrm{J}}=\beta_{1} \pi a_{0}^{2} I_{\max } / \alpha_{1}\left(\alpha_{1}+\beta_{1}\right)$ and we have [12-14]

and

$$
f(r)=\left\{\begin{array}{lll}
1, & \text { if } & 0 \leqslant r \leqslant a_{0} \\
0 & \text { if } & a_{0}<r<\infty
\end{array}\right.
$$

$$
f(r)=\mathrm{e}^{-\left(r / a_{0}\right)^{2}}
$$

to account for the non-Gaussian and Gaussian nature of the laser beam, respectively.

The resulting thermal field $\theta=T-T_{0}=\theta(r, z, t)$ was derived using Hankel transformation and Bessel series expansion technique [22]. The temperature distribution is given analytically in the Appendix. Figure 4 shows the histories of the temperature rise in the centers of the front and rear surfaces, respectively. Also, the experimental results are given in the figure [22]. The good agreement of numerical results with the test can be seen in the figure. Figure 5 shows the variations of the temperature rise with $z / a_{0}$ in the center of the brass foil. As would be expected, a high temperature state is built on the specimen front surface impinged by a normal incident laser beam. This means that the temperature gradient in the $z$-direction, i.e. $\partial \theta / \partial z$ is high at the earlier stage of laser irradiation, and 

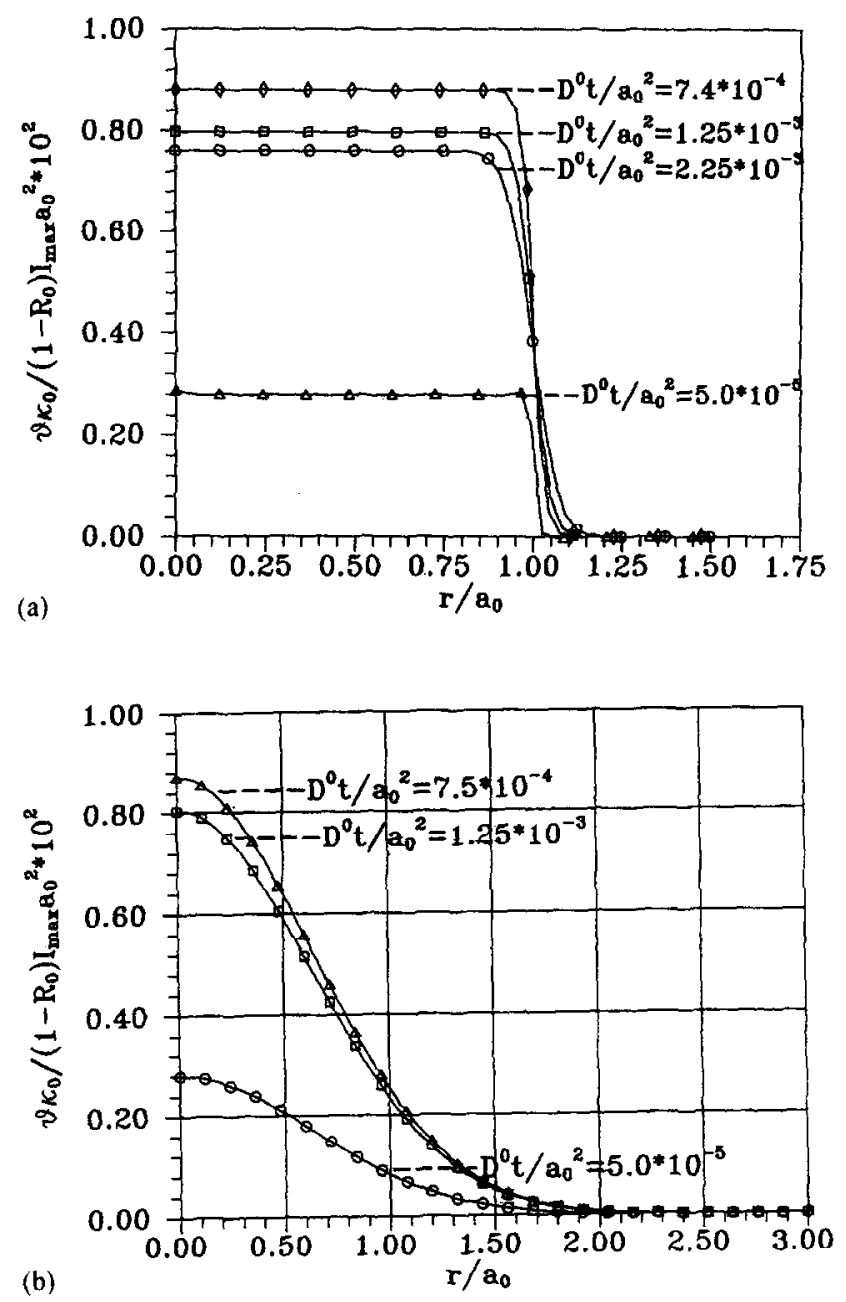

Fig. 6. Variations of dimensionless temperature rise with $r / a_{0}$ on the front surface, where the spatial shape of the laser is non-Gaussian (a) and Gaussian (b) type.

then gradually decays. At the late stage of laser irradiation, the temperature gradient tends to zero. This temperature gradient plays a key role in leading to the brass foil bulging.

The spatial profile of temperature distribution is influenced definitely by the spatial shape of the laser beam, as shown in Fig. 6(a) and (b). Therefore, different spatial shapes of laser beam lead to different temperature distributions even though the net energy and duration are the same. For a non-Gaussian type laser as used in the test, the spatial distribution of the temperature is uniform within the laser irradiated region and declines very sharply towards the edge where the laser spot terminates. The steep temperature gradient prevails across the periphery of the laser where the spot terminates. As we can see in the following section this causes that high shear deformation occurs around a rim near the outer edge of the laser beam.

\subsection{Solution of the non-linear coupled equations}

When the material parameters and the temperature rise $\theta$ are known, the governing equations (20)-(22) for the deflection and the shear deformation can be solved for the plate with a clamped edge which is restrained from radial movement.

$$
u(0)=u(a)=0, \quad w^{\prime}(0)=w(a)=w^{\prime}(a)=0, \quad \phi(0)=\phi(a)=0
$$

From equation (20) and the boundary condition $u(0)=u(a)=0$, we obtain

$$
\frac{\mathrm{d}(r u)}{\mathrm{d} r}=\frac{1}{h} \alpha r(1+v) p_{\mathrm{T}}+c^{*} \frac{r}{D}-\frac{1}{2} r w^{\prime 2}
$$


where

$$
c^{*}=\left[\frac{1}{2} \int_{0}^{a} r w^{\prime 2} \mathrm{~d} r-\frac{1}{h} \int_{0}^{a} \alpha r(1+v) p_{\mathrm{T}} \mathrm{d} r\right] /\left(\int_{0}^{a} \frac{r}{D} \mathrm{~d} r\right)
$$

Substituting equations (27) and (28) into (21), we obtain

$$
\begin{gathered}
\nabla^{2}\left(D \nabla^{2} w\right)-\frac{12}{h^{2}} c^{*} \nabla^{2} w-\frac{1}{r}\left\{\frac{\mathrm{d}^{2}}{\mathrm{~d} r^{2}}[(1-v) D] w^{\prime}+\frac{\mathrm{d}}{\mathrm{d} r}[(1-v) D] w^{\prime \prime}\right\} \\
+\frac{12}{h^{3}}\left\{D \alpha(1+v) \nabla^{2} m_{\mathrm{T}}+m_{\mathrm{T}} \nabla^{2}[D \alpha(1+v)]+2 m_{\mathrm{T}}^{\prime} \frac{\mathrm{d}}{\mathrm{d} r}[D \alpha(1+v)]\right\} \\
-\frac{4}{5}\left\{\nabla^{2}(D \phi)+\phi^{\prime} \frac{\mathrm{d}}{\mathrm{d} r}[D(v-1)]+\phi \frac{\mathrm{d}^{2}}{\mathrm{~d} r^{2}}[D(v-1)]\right\}=0
\end{gathered}
$$

Now, the governing equations (22) and (29) for the unknown variables $\phi$ and $w$ are the new non-linear coupled equations of shear strains and large deflections with the corresponding boundary conditions (26). They are solved with the help of the Galerkin method and the iterative method. The approximate expression for $w$ is selected directly from the linear theory of plates with small deflections, and is

$$
w=\sum_{m=1}^{8} c_{m}\left(1-\frac{r^{2}}{a^{2}}\right)^{m+1}
$$

By using the Galerkin method, the unknown coefficients $c_{m}(m=1,2, \ldots, 8)$ can be obtained from the following non-linear algebraic equations.

$$
\begin{aligned}
& \int_{0}^{a} r \mathrm{~d} r\left(\nabla^{2}\left(D \nabla^{2} w\right)-\frac{12}{h^{2}} c^{*} \nabla^{2} w-\frac{1}{r}\left\{\frac{\mathrm{d}^{2}}{\mathrm{~d} r^{2}}[(1-v) D] w^{\prime}+\frac{\mathrm{d}}{\mathrm{d} r}[(1-v) D] w^{\prime \prime}\right\}\right. \\
& +\frac{12}{h^{3}}\left\{D \alpha(1+v)\left(\nabla^{2} m_{\mathrm{T}}+p_{\mathrm{T}} \nabla^{2} w\right)+m_{\mathrm{T}} \nabla^{2}[D \alpha(1+v)]+2 m_{\mathrm{T}}^{\prime} \frac{\mathrm{d}}{\mathrm{d} r}[D \alpha(1+v)]\right\} \\
& \left.-\frac{4}{5}\left\{\nabla^{2}(D \phi)+\phi^{\prime} \frac{\mathrm{d}}{\mathrm{d} r}[D(v-1)]+\phi \frac{\mathrm{d}^{2}}{\mathrm{~d} r^{2}}[D(v-1)]\right\}\right)\left[1-\left(\frac{r}{a}\right)^{2}\right]^{m+1} \mathrm{~d} r=0
\end{aligned}
$$

The non-linear coupled equations of shear strains and large deflections can be solved with the iterative method. First, we can solve the coefficient $c_{m}$ from the non-linear algebraic equations (31) with $\phi=0$. As long as $w$ is obtained from (30) and (31), the unknown variable $\phi$ can be solved from the two order differential equation (22) with the clamped boundary conditions $\phi(0)=\phi(a)=0$. Then, when the unknown variable $\phi$ is obtained from equation (22), the coefficient $c_{m}(m=1,2,3, \ldots, 8)$ can again be obtained by solving the non-linear algebraic equations (31) with $\phi \neq 0$. Based on these iterative methods, the numerical results are obtained for deflections and the average shear strains are obtained at different times.

\section{NUMERICAL RESULTS AND DISCUSSIONS}

To analyse the shear deformations and the large deflections for the reverse bulging and plugging in brass foil induced by a long pulsed laser, we assume that Young's modulus varies according to the temperature $T$. From the experimental test of Young's modulus of brass [23], $E(T)$ is given by

$$
E(T)=E_{0} \sum_{i=0}^{5} d_{i}\left(\frac{T}{\bar{T}}-1\right)^{i}
$$

where $E_{0}, d_{i}$ and $\bar{T}$ are constants. The Poisson ratio $v$ and the thermal expansion coefficient $\alpha$ are assumed to be constant. The material constants and the laser parameters are listed in Table 1. From equation (5), the average shear strain is $\gamma=\frac{1}{3} \phi$.

The numerical results for the deflection $w$ and average shear strain $\gamma$ will be presented graphically for various cases. Case 1 corresponds to the classical Kirchhoff-Love plate theory, i.e. $D=D_{0}, \phi=0$ and eliminating the term $\left(w^{\prime}\right)^{2}$ in (3). In this case, equation (29) 
Table 1. Material constants and laser parameters

\begin{tabular}{lccc}
\hline & Material constants & Laser parameters \\
\hline$E_{0}=8.23 \times 10^{6} \mathrm{~N} / \mathrm{cm}^{2}$ & $d_{0}=1.0$ & $d_{5}=0.0202$ & $\alpha_{1}=1.5 \times 10^{4} \mathrm{~s}^{-1}$ \\
$C_{\mathrm{p}}=0.374 \mathrm{~J} / \mathrm{g} \mathrm{K}$ & $d_{1}=-0.35$ & $T=510 \mathrm{~K}$ & $\beta_{1}=8.0 \times 10^{4} \mathrm{~s}^{-1}$ \\
$k_{0}=1.09 \mathrm{~W} / \mathrm{cm} \mathrm{K}$ & $d_{2}=-0.127$ & $v=0.33$ & $a_{0}=0.25 \mathrm{~cm}$ \\
$\rho=8.7 \mathrm{~g} / \mathrm{cm}^{3}$ & $d_{3}=-0.0205$ & $a=0.5 \mathrm{~cm}$ & $R_{0}=0.9$ \\
$\alpha=1.7 \times 10^{-5} / \mathrm{K}$ & $d_{4}=-1.566 \times 10^{-3}$ & $h=0.01 \mathrm{~cm}$ & \\
\hline
\end{tabular}

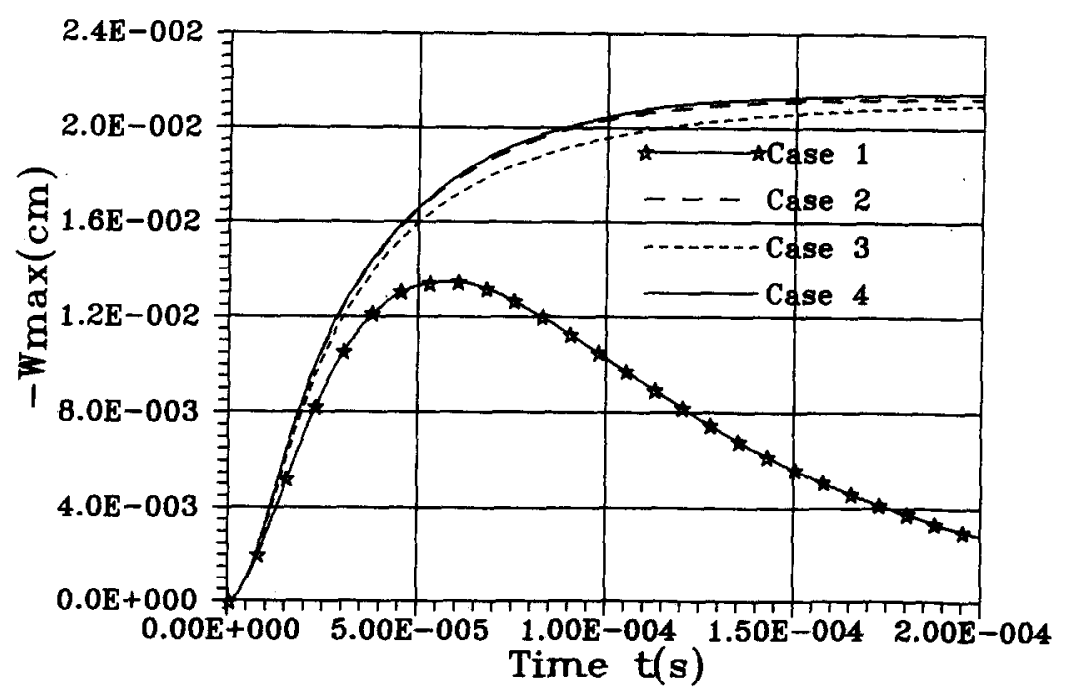

Fig. 7. Maximum deflections vs time for a non-Gaussian type laser beam and $E_{\mathrm{J}}=10 \mathrm{~J}$ for various cases: case $1-D=D_{0}, \phi=0$ and eliminating the term $w^{\prime 2}$ in (3); case $2-D \neq 0, \phi=0$; case $3-D=D_{0}, \phi \neq 0$; case $4-D \neq D_{0}, \phi \neq 0$.

is reduced to:

$$
\nabla^{4} w+\frac{12}{h^{3}} \alpha(1+v) \nabla^{2} m_{\mathrm{T}}=0
$$

Case 2 corresponds to the large deflection theory of Ohnabe and Mizuguchi [18] without consideration of the effect of shear deformation $\phi$ on the deflection $w$, i.e. $D \neq D_{0}$ and $\phi=0$. Case 3 corresponds to the results obtained from the new non-linear coupled equations of deflection and shear deformation, but without consideration of the dependence of Young's modulus on temperature, i.e. $D=D_{0}, \phi \neq 0$. Case 4 corresponds to the numerical results obtained from the new equations (22) and (31) derived in the present work.

\subsection{Deflection}

Figure 7 shows the histories of maximum deflection with a non-Gaussian type of laser beam and $E_{\mathrm{J}}=10 \mathrm{~J}$ for the various cases discussed in the above. The corresponding transient deflection distributions to the histories of maximum deflection of Fig. 7 are shown in Fig. 8. From these figures, one can see that: (a) the target sheet subjected to laser heating does bulge in the direction opposite to the incident laser beam; (b) only the deflection in case 1 tends to zero when the time is long enough, but the delections in the other cases always increase with the increasing of time; (c) although the deflection in case 3 is constantly less than that in case 2, the contributions of the dependence of Young's modulus on temperature and the shear deformation to the deflection are almost the same, so the effects of $E(T)$ and $\gamma$ on the deflection are all non-linear.

To express the effect of absorbed laser energy by the target on the deflection, Fig. 9 shows the histories of the maximum deflection in cases 1 and 2 with $E_{\mathrm{J}}=10 \mathrm{~J}$ and $E_{\mathrm{J}}=15 \mathrm{~J}$. Because of the dependence of Young's modulus on temperature, the relationships of deflection with the absorbed laser energy $E_{\mathrm{J}}$ are also non-linear. The non-linear relationship of deflection with laser energy $E_{\mathrm{J}}$ can obviously be observed from Fig. 9. 


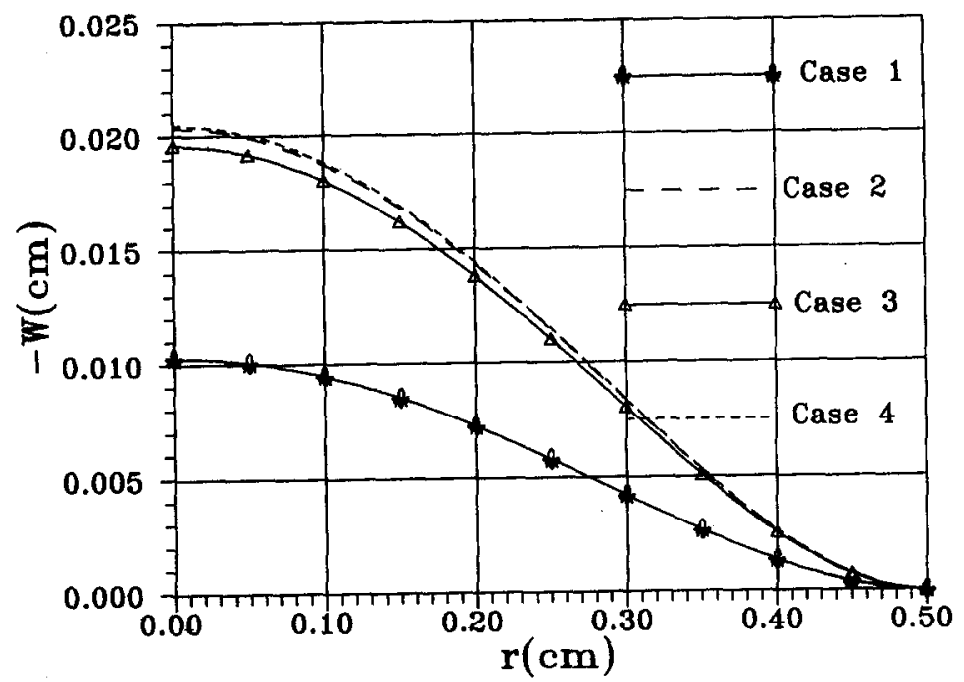

Fig. 8. Transicnt deflection distributions at $t=100 \mu \mathrm{s}$ for a non-Gaussian type laser bcam and $E_{\mathrm{J}}=10 \mathrm{~J}$ for various cases: case $1-D=D_{0}, \phi=0$ and eliminating the term $w^{\prime 2}$ in (3); case $2-D \neq 0, \phi=0$; case $3-D=D_{0}, \phi \neq 0$; case $4-D \neq D_{0}, \phi \neq 0$.

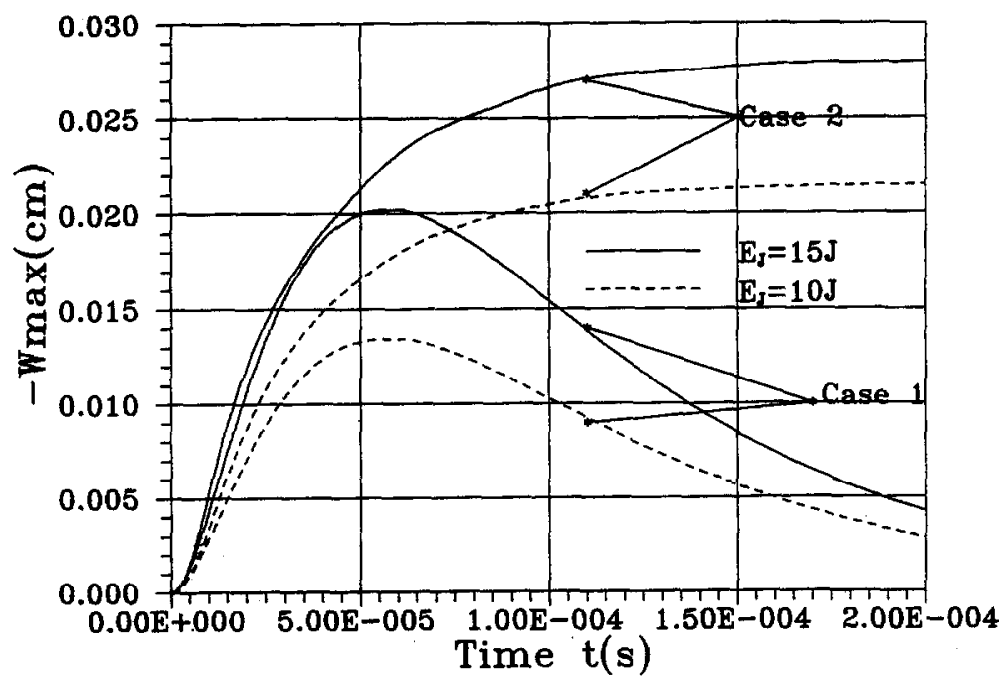

Fig. 9. The curves of maximum deflections vs time for the laser beam with non-Gaussian type and $E_{\mathrm{J}}=10 \mathrm{~J}, E_{\mathrm{J}}=15 \mathrm{~J}$.

\subsection{Shear deformation}

The numerical results of shear deformation are discussed in cases 3 and 4. Figure 10 shows the transient average shear strain $\gamma$ vs the radial coordinate for the non-Gaussian and Gaussian types of laser beam with $E_{\mathrm{J}}=10 \mathrm{~J}$ at different times in case 3 . The histories of the maximum average shear strain $\gamma$ are shown in Fig. 11 for the non-Gaussian type laser beam with $E_{\mathrm{J}}=10 \mathrm{~J}$ and $E_{\mathrm{J}}=15 \mathrm{~J}$ in case 3 . Note that the shear strains in case 3 tend to zero when the time is long enough. Figure 12 shows the transient average shear strain $\gamma$ distributions with $E_{\mathrm{J}}=10 \mathrm{~J}$ at different time in case 3 for the non-Gaussian and Gaussian type laser beam.

It is observed from Fig. 10 that only the shear strain $\gamma$ is not zero within the laser spot edge region. This important result confirms the experimental observations, i.e. the existence of large shear strain $\gamma$ within the laser spot edge region [12-14]. From the comparison of the shear strain distribution induced by a non-Gaussian laser beam and by a Gaussian laser beam (Fig. 12), one can conclude that the former offers a formidable potential for the new type of failure by plugging, however, the latter has a little potential for the new type of failure by plugging. 


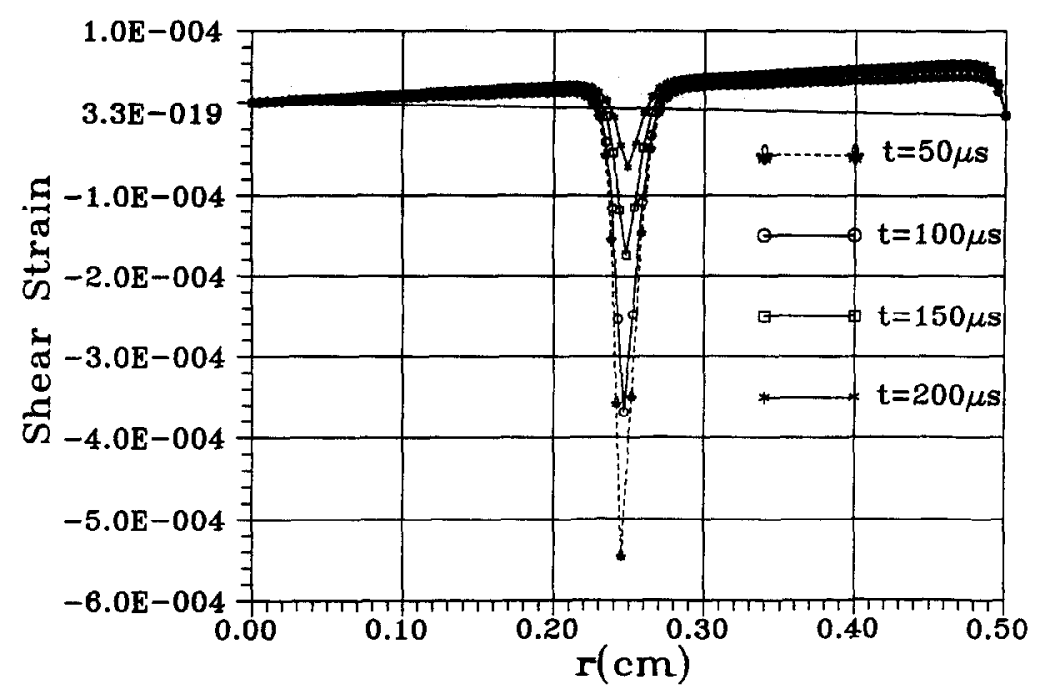

Fig. 10. Transient average shear strain $\gamma$ vs the radial coordinate for the non-Gaussian type laser beam with $E_{J}=10 \mathrm{~J}$ at different times in case 3 . Note that the maximum shear strain appears within the laser spot edge region.

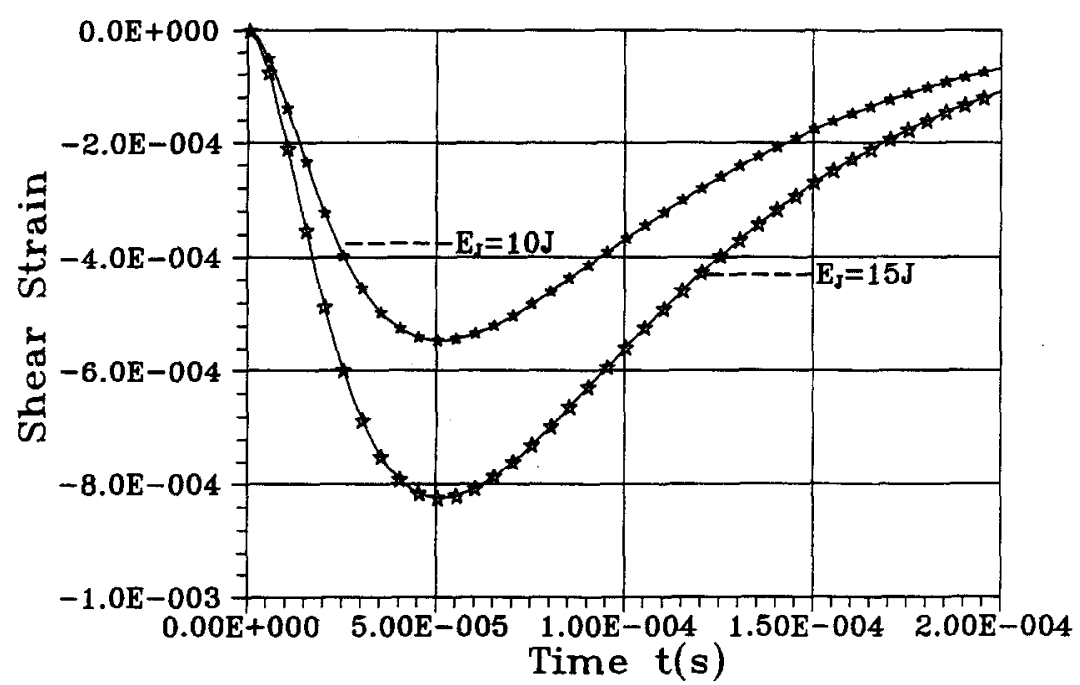

Fig. 11. The curves of the maximum average shear strain $\gamma$ vs time for the non-Gaussian type laser beam with $E_{\mathrm{J}}=10 \mathrm{~J}$ and $E_{\mathrm{J}}=15 \mathrm{~J}$ in case 3 .

This reveals that the spatial shapes of a laser beam, indeed, play an important role in controlling the damage types. The spatial structure effect is also important in laser processing of materials $[24,25]$. Hector and Hetnarski [24] studied the thermal stress field in an elastic half-space due to a single pulse from a laser for the general case of a mixedmode structure beam. The investigations $[24,25]$ show that the doughnut mode structure is useful for selected heat treating, cutting and welding applications while the Gaussian mode structure is employed in the vast majority of laser cutting applications.

\subsection{The effect of $E(T)$ on shear deformation}

The transient average shear strain distributions are shown in Fig. 13 for the nonGaussian type laser beam with $E_{\mathrm{J}}=10 \mathrm{~J}$ at different times in case 4 . Also, Fig. 14 shows the histories of the maximum average shear strain for a non-Gaussian type of laser beam with $E_{\mathrm{J}}=10 \mathrm{~J}$ in case 4 . The value of the shear strain in case 3 is always negative, but the dependence of Young's modulus on temperature will always yield positive shear strain (Figs 13 and 14). The two order differential equation (22) about the variable $\phi$ can be written 


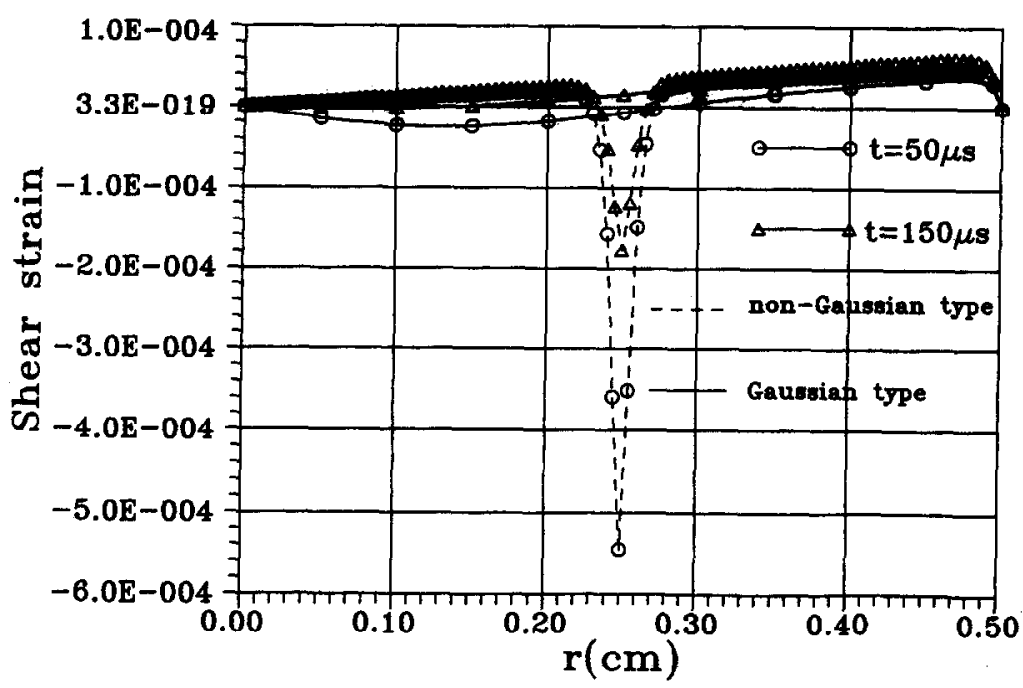

Fig. 12. Transient average shear strain $\gamma$ vs the radial coordinate for the non-Gaussian and Gaussian types of laser beam with $E_{\mathrm{J}}=10 \mathrm{~J}$ at different times in case 3.

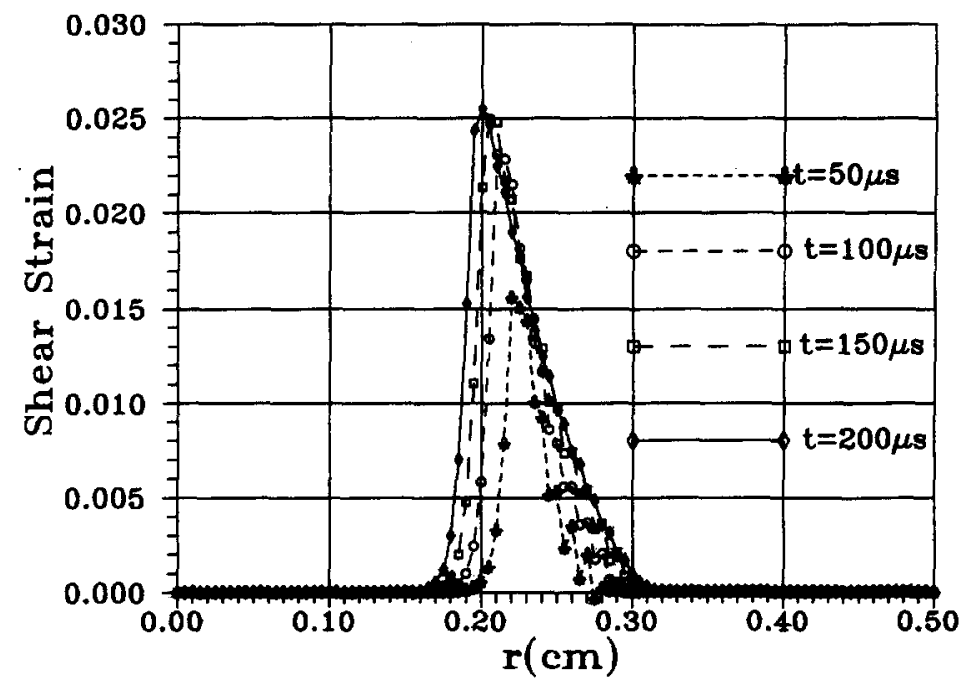

Fig. 13. Transient average shear strain distributions vs the radial coordinate for the non-Gaussian type of laser beam with $E_{\mathrm{J}}=10 \mathrm{~J}$ at different times in case 4 .

again in the following form

$$
\begin{gathered}
\frac{17}{21} D \phi^{\prime \prime}+\frac{17}{21}\left(\frac{D}{r}+D^{\prime}\right) \phi^{\prime}-\left[\frac{17}{21} \frac{1}{r}\left(\frac{D}{r}-v D^{\prime}\right)+\frac{4}{h^{2}}(1-v) D\right] \phi \\
-\frac{\mathrm{d}}{\mathrm{d} r}\left[D \nabla^{2} w+\frac{15}{h^{3}} D \alpha(1+v)\left(m_{\mathrm{T}}-n_{\mathrm{T}}\right)\right]-w^{\prime} \frac{1}{r}(v-1) D^{\prime}=0
\end{gathered}
$$

From the above equation, the variable coefficient of $\phi$ is $-\left[\frac{17}{21}(1 / r)\left(D / r-v D^{\prime}\right)+\right.$ $\left.\left(4 / h^{2}\right)(1-v) D\right]$. In case $3, D=D_{0}$, i.e. $D^{\prime}=0$, the coefficient $-\left[\frac{17}{21}(1 / r)\left(D / r-v D^{\prime}\right)+(4 /\right.$ $\left.\left.h^{2}\right)(1-v) D\right]$ is constantly negative. In case $4, D \neq 0, D^{\prime}=(\mathrm{d} D / \mathrm{d} T)(\mathrm{d} T / \mathrm{d} r)>0$. The $D^{\prime}$ is so large within the laser spot edge region that the coefficient $-\left[\frac{17}{21}(1 / r)\left(D / r-v D^{\prime}\right)+(4 /\right.$ $\left.\left.h^{2}\right)(1-v) D\right]$ is positive. The change of the coefficient from a negative value in case 3 to a positive value in case 4 results in the change of the shear strain from a negative value in case 3 to a positive one in case 4 . The average shear strain in case 4 is much larger than that in case 3. This means that the shear deformation comes under the strong influence of the dependence of the Young's modulus $E(T)$ on temperature $T$. The shear strain in case 4 constantly increases with the increasing of time, this is not like that in case 3. 


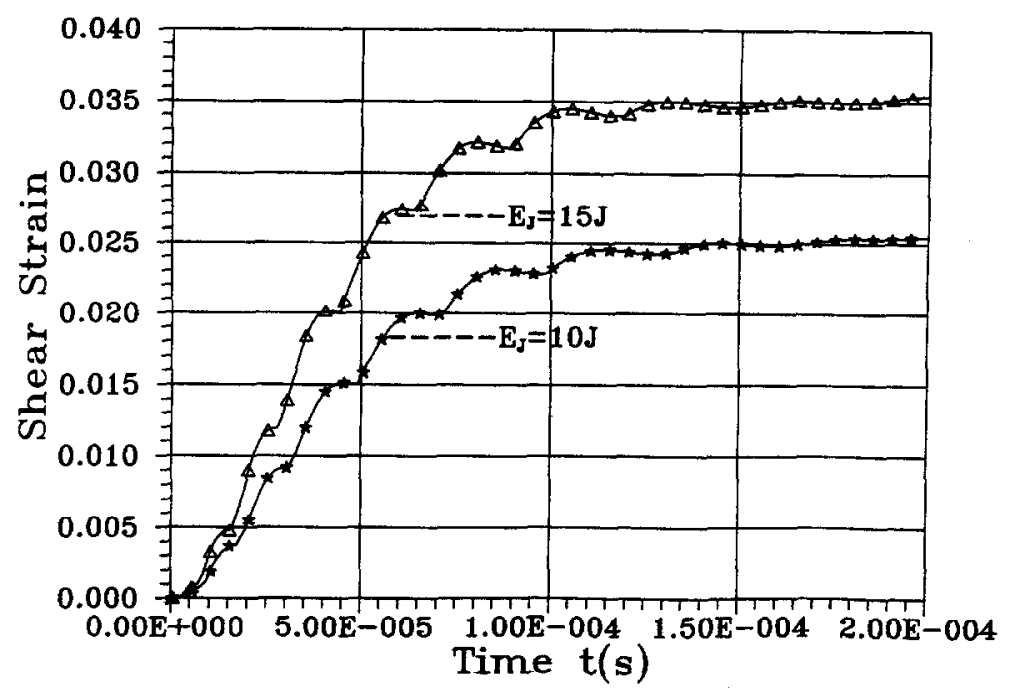

Fig. 14. The curves of the maximum average shear strain vs time for the non-Gaussian type laser beam with $E_{\mathrm{J}}=10 \mathrm{~J}$ and $E_{\mathrm{J}}=15 \mathrm{~J}$ in case 4 .

\section{CONCLUSIONS}

In the present article, we have derived the new coupled governing equations of shear deformation and large deflections of a heated, non-homogeneous circular plate. The governing equations are obtained based on the large deflections of Berger [17], Ohnabe and Mizuguchi [18] and the parabolic shear deformation theory of Bhimaraddi and Stevens [20]. We use the new coupled governing equations to analyse the failure mode induced by laser. The new non-linear coupled equations were solved by employing the Galerkin and iterative methods.

Numerical results of the deflection and the average shear strain for various spatial shape laser beams are shown in the graphs. It was found that large shear strain does exist within the laser spot edge region for a non-Gaussian laser beam. These important conclusions are in good agreement with the experimental observations [12-14]. The non-Gaussian laser beam has a formidable potential for the new failure mode, however, the Gaussian laser beam has little potential for the new failure mode. It was also found that $D(T)$ had important influence on the large deflections and the shear strain.

Acknowledgements-Support for this research program was provided by the Field of Laser Technology, 863NHT Research Development Program. The research is also supported by the National Natural Science Foundation of China and the Education Commission of Hunan Province. Their support is gratefully acknowledged. The authors show their sincere gratitude to the referees for their careful proof-reading and many valuable suggestions.

\section{REFERENCES}

1. J. F. Ready, Effect of High-power Laser Radiation. Academic Press (1971).

2. M. von Allmen, Laser Beam Interactions with Materials. Springer-Verlag (1987).

3. B. C. H. Wendlant, Theoretical studies of the interaction of high-power laser beam and metals, NASA-74$16188(1973)$.

4. A. Kar and J. Mazumder, Two-dimensional model for material damage due to melting and vaporization during laser irradiation. J. Appl. Phys. 68 (8), 3884-3891 (1990).

5. J. A. Fox and D. N. Barr, Laser-induced shock effects in plexiglass and 6061-T6 aluminum. Appl. Phys. Lett. 22 (11), 594-596 (1973).

6. J. Hermann, C. Boulmer-Leborgne, B. Dubreuil and I. N. Mihailescu, Influence of irradiation conditions on plasma evolution in laser-surface interaction. J. Appl. Phys. 74 (5), 3071-3079 (1993).

7. S. Eleizer, Gilath 1. and T. Bar-Noy, Laser induced spall in metals: experiment and simulation. J. Appl. Phys. 67 (2), 715-724 (1990).

8. M. Boustie and F. Cottet, Experimental and numerical study of laser induced spallation into aluminium and copper targets. J. Appl. Phys. 69 (11), 7533-7538 (1991).

9. A. D. Zweig, A thermo-mechanical model for laser ablation. J. Appl. Phys. 70 (3), 1684-1691 (1991).

10. C. L. Chan and J. Mazumder, One dimensional steady-state model for damage by vaporization and liquid expulsion due to laser-material interaction. J. Appl. Phys. 62 (11), 4579-4586 (1987). 
11. J. E. Medford and P. M. Gray, The response of structural materials to combined laser and mechanical loading, AIAA-80-1550 (1980).

12. Z. P. Duan, Y. C. Zhou et al., On laser induced reverse plugging effect. Proceedings of IUT AM Symp. on Impact Dyn. (Edited by Z. M. Zheng et al.), pp. 176-186.

13. Y. C. Zhou and Z. P. Duan, Circular brass foil failed by plugging opposite to the direction of incident laser beam. Theoretical and Applied Fracture Mechanics 24 (2), 135-138 (1996).

14. Y. C. Zhou, Z. P. Duan and B. M. Xie, Long pulsed laser induced reverse bulging and plugging. Int. J, Engng Sci. (in press).

15. C. E. Anderson and S. R. Bodner, Ballistic impact: the status of analytical and numerical modeling. Int. J. Impact Engng 7 (1), 9-35 (1988).

16. M. Shoukry, S. Nair and S. Kalpakjian, Effect of shear deformation on the dynamic plastic bending of metallic plates during normal penetration. Int. J. Engng Sci. 29 (9), 1035-1052 (1991).

17. H. M. Berger, A new approach to the analysis of large deflections of plates. J. Appl. Mech. 22 (3), 465-472 (1965).

18. H. Ohnabe and F. Mizuguchi, Large deflections of heated non-homogeneous circular plates with radially varying rigidity. Int. J. Non-Linear Mech. 28 (4), 365-372 (1993).

19. A. Bhimaraddi and $K$. Chandrashekhara, Non-linear vibrations of heated antisymmetric angle-ply laminated plates. Int. J. Solids Structures 30 (9), 1255-1268 (1993).

20. A. Bhimaraddi and L. K. Stevens, A higher order theory for free vibration of orthotropic, homogeneous, and laminated rectangular plates. J. Appl. Mech. 51 (1), 195-198 (1984).

21. B. A. Boley and J. H. Weiner, Theory of Thermal Stress. Wiley (1960).

22. Y. C. Zhou, Z. P. Duan and Q. B. Yang, A thermal-elastic analysis on laser-induced reverse bulging and plugging in circular brass foil. Int. J. Solids and Structures (in press).

23. The Group of Handbook of Chinese Aeronautic Materials, Handbook of Chinese Aeronautic Materials (in Chinese). Chinese Criterion Press (1989).

24. L. G. Hector Jr. and R. B. Hetnarski, Thermal stresses due to a laser pulse: elastic solution. J. Appl. Mech. 63 (1), 38-46 (1996).

25. J. Powell, $\mathrm{CO}_{2}$ Laser Cutting. Springer-Verlag, New York (1993).

\section{APPENDIX}

In this Appendix, the temperature rise distribution is expressed as the following formula.

$$
\begin{aligned}
\theta & =\frac{\left(1-R_{0}\right) a_{0} I_{\max }}{k_{0}} \sum_{k_{n}} \frac{2 J_{0}\left(k_{n} \frac{r}{a_{0}}\right) f^{*}\left(k_{n}\right)}{a_{0}\left(\frac{a}{a_{0}}\right)^{2} J_{1}^{2}\left(k_{n} \frac{a}{a_{0}}\right)}\left\{\frac{1}{2} g(t)\left(\frac{z}{a_{0}}-\frac{h}{2 a_{0}}\right)^{2}\right. \\
& -6\left(\frac{h}{a_{0}}\right)^{2} g(t)+\frac{\mathrm{e}^{-a_{1} t}-\mathrm{e}^{-k_{n}^{2} D^{o} t / a_{\theta}^{2}}}{k_{n}^{2}-\alpha_{1} a_{0}^{2} / D^{0}}-\frac{\mathrm{e}^{-\left(\alpha_{1}+\beta_{1}\right) t}-\mathrm{e}^{-k_{n}^{2} D^{0} t / a_{0}^{2}}}{k_{n}^{2}-\left(\alpha_{1}+\beta_{1}\right) a_{0}^{2} / D^{0}} \\
& -2 \sum_{m=1}^{\infty}\left(\frac{h}{m \pi a_{0}}\right)^{2}\left[g(t)-\left(\frac{m \pi a_{0}}{h}\right)^{2} \frac{\mathrm{e}^{-\alpha_{1} t}-\mathrm{e}^{-k_{n}^{2} D^{\circ} t / a_{0}^{2}} \mathrm{e}^{-\left(m \pi a_{0} / h\right)^{2} t}}{k_{n}^{2}+\left(\frac{m \pi a_{0}}{h}\right)^{2}-\alpha_{1} a_{0}^{2} / D^{0}}\right. \\
& \left.\left.+\left(\frac{m \pi a_{0}}{h}\right)^{2} \frac{\mathrm{e}^{-\left(z_{1}+\beta_{1}\right) t}-\mathrm{e}^{-k_{n}^{2} D^{\circ} / / a_{0}^{2}} \mathrm{e}^{-\left(m \pi a_{0} / h\right)^{2} t}}{k_{n}^{2}+\left(\frac{m \pi a_{0}}{h}\right)^{2}-\left(\alpha_{1}+\beta_{1}\right) a_{0}^{2} / D^{0}}\right] \cos \left[\frac{m \pi}{h}\left(z+\frac{h}{z}\right)\right]\right\}
\end{aligned}
$$

where $k_{0}, D^{0}=k_{0} / \rho C_{\mathrm{p}}$ are the thermal conductivity, thermal diffusion coefficient, respectively, and $R_{0}$ is the reflection coefficient of laser beam. $k_{n}$ are the roots of equations $J_{0}\left(k_{n} a / a_{0}\right)=0, J_{n}(x)$ are the $n$th Bessel functions of the first kind. The transform coefficient $f^{*}\left(k_{n}\right)$ is given by

$$
f^{*}\left(k_{n}\right)=\int_{0}^{a} f(r)\left(J_{0} k_{n} r / a_{0}\right) \frac{r}{a_{0}^{2}} \mathrm{~d} r .
$$

